\title{
Description de deux espèces nouvelles du genre Lychnus (Mollusca, Gastropoda, Anadromidae) au Rognacien (Maastrichtien supérieur) de Vitrolles (Bouches-du-Rhône, France)
}

\author{
Guy Turin* \\ 8 rue de la Mairie, 77320 Saint-Martin-du-Boschet, France
}

\begin{abstract}
Résumé - Sur la commune de Vitrolles (Bouches-du-Rhône), le Rognacien (Maastrichtien supérieur) calcaire surplombant la partie orientale de l'étang de Berre comprend principalement la barre calcaire de Rognac (Rognacien moyen) avec des niveaux marneux intermédiaires, suivi de $12 \mathrm{~m}$ au-dessus d'une cuesta calcaire attribuée au Rognacien supérieur. Le genre Lychnus décrit par Matheron en 1832, comprend dans ce Rognacien terminal deux espèces Lychnus matheroni Requien, 1842 et L. vitrollensis Répelin, 1920, ces taxons sont accompagnés par deux nouvelles espèces Lychnus siruguei nov. sp. et Lychnus applanatus nov. sp. Ces deux nouvelles espèces n'ont jamais été décrites, ni figurées, elles font l'objet de la présente publication.
\end{abstract}

Mots clés : Vitrolles / Rognacien supérieur / Maastrichtien / Lychnus / taxons / nouvelles espèces

\begin{abstract}
Description of two new species on the genus Lychnus (Mollusca, Gastropoda, Anadromidae) the Rognacien (upper Maastrichtian) of Vitrolles (Bouches-du-Rhône, French). On the commune of Vitrolles territory (Bouches-du-Rhône) the calcareous Rognacien (upper Maastrichtian) above the eastern part of the Étang de Berre consists mainly of the Rognac calcareous bar (middle Rognacien) with intermediary marly layers topped $12 \mathrm{~m}$ above by a calcareous cuesta attributed to the upper Rognacien. The genus Lychnus described by Matheron in 1832 consists in this terminal Rognacien two species Lychnus matheroni Requien, 1842 and the L. vitrollensis Répelin, 1920, these taxa are accompanied by two new species Lychnus siruguei nov. sp. and Lychnus applanatus nov. sp. These two new species, which had never been described or sketched until now, are the subject of this publication.
\end{abstract}

Keywords: Vitrolles / upper Rognacien / Maastrichtian / Lychnus / taxa / new species

\section{Introduction}

Si la présence du genre Lychnus en Provence est connue depuis le $\mathrm{XIX}^{\mathrm{e}}$ siècle (Matheron, 1832), il est certain qu'à l'exception de travaux plus récents (Haug, 1908-1911; Repelin, 1920 ; Fabre-Taxy, 1959), le complément du matériel récolté n'a pratiquement jamais fait l'objet de publications paléontologiques comportant des descriptions, coupes et photographies. Le présent travail consiste à enrichir la biodiversité de ce genre en créant deux nouvelles espèces dans le Rognacien supérieur (Maastrichtien supérieur), car à ma connaissance jusqu'à maintenant, aucune espèce du genre Lychnus n'a été récoltée dans les couches sus-jacentes de la

*Auteur correspondant : turin.guy77@orange.fr partie sommitale du calcaire de Rognac (Rognacien moyen) (Repelin, 1899). Les collections du Muséum d'Histoire Naturelle de Marseille et celle du MNHN de Paris, sont dépourvues également de ces nouvelles espèces.

\subsection{Cadres géographique}

Les espèces nouvelles Lychnus siruguei nov. sp. et $L$. applanatus nov. sp., ont été récoltées sur la commune de Vitrolles (Fig. 1), au chemin du Trou du Loup, aux coordonnées: 43. 469092, 5. 243445, dans un banc calcaire carbonaté $\left(n^{\circ} 100\right.$, Fig. 2$)$. Ce niveau se prolonge au sud, vers le Griffon et la commune des Pennes-Mirabeau, vers le nord on le retrouve à Saragousse sur la commune de Rognac. Cet horizon calcaire est totalement absent dans le Rognacien supérieur de la partie orientale du bassin d'Aix (Dughi et Sirugue, 1958). 


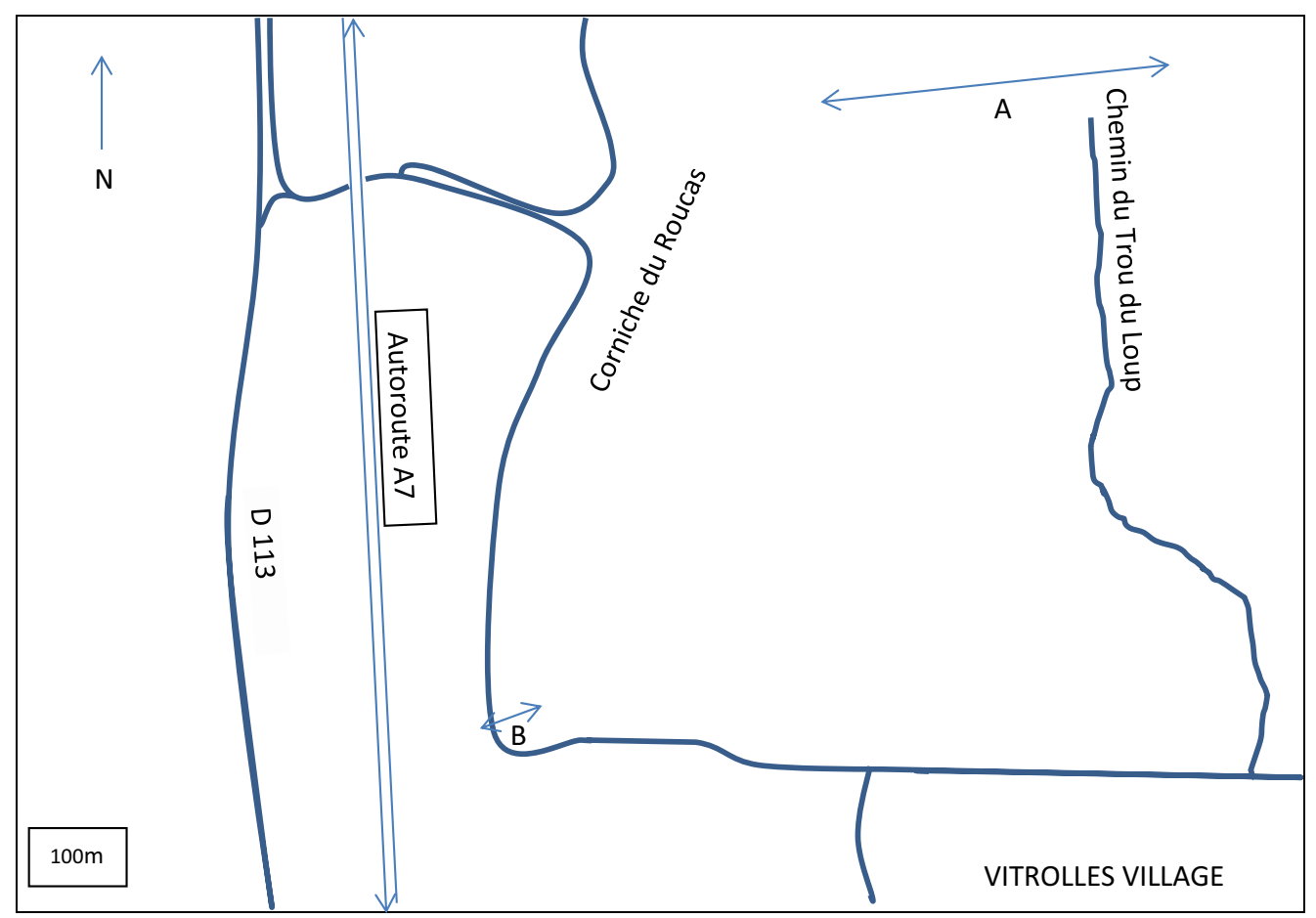

Fig. 1. Localisation des deux coupes A et B sur la commune de Vitrolles (Bouches-du-Rhône).

\subsection{Lithologie et biostratigraphie}

Les deux nouvelles espèces ont été recensées $12 \mathrm{~m}$ audessus du sommet de la cuesta du calcaire de Rognac (Rognacien moyen), sur la partie sommitale d'une barre calcaire de $4 \mathrm{~m}$ d'épaisseur. Celle-ci est constituée sur les deux premiers mètres d'une zone de calcaire gris marneux sans fossiles de gastéropodes, suivie sur les deux mètres suivant d'un calcaire carbonaté rose comprenant les espèces décrites dans le tableau de la coupe A (Fig. 2). L'horizon sus-jacent est composé de $0,5 \mathrm{~m}$ de calcaire marneux rose, c'est le dernier banc fossilifère qui a livré un Lychnus matheroni Requien, 1842 (Requien, 1842), du Maastrichtien supérieur de la série fluvio-lacustre et continentale de Vitrolles. Concernant L. matheroni Requien, d'Orbigny a changé la dénomination de ce genre par Tomogeres en 1847 (d'Orbigny, 1850; Matheron, 1862). La puissante série d'argiles de couleur orangé (couche $n^{\circ} 102$, Fig. 2) comprise entre ce dernier banc calcaire à Lychnus et le marbre de Vitrolles n'a livrée aucun fossile de gastéropodes, cette zone est encore attribuée au Crétacé supérieur car le calcaire ou marbre de Vitrolles susjacent constitue la partie basale du Danien.

En ce qui concerne le Rognacien moyen, une coupe B a été réalisée sur la corniche du Roucas (Fig. 3), elle comprend la totalité du calcaire de Rognac avec des niveaux marneux et ligniteux intermédiaires. Ces bancs de calcaire lacustre font 22,5 m d'épaisseur sur la totalité, la série ici est beaucoup plus mince que sur la commune de Rognac (Durand, 1978), cette matière massive de couleur gris blanc est composée de Chara et Cyanophycées, les horizons marneux déposés entre-deux représentent les périodes d'asséchement d'un ancien lac. Le genre Lychnus est présent surtout dans la partie sommitale de cette série, les espèces récoltées sont les suivantes: Lychnus vidali Répelin, 1920, L. urgonensis Matheron, 1843, (=L. bourguignati Munier-Chalmas, 1885), L. matheroni Requien, 1842, L. ellipticus Matheron, 1832 et $L$. rimatus Matheron, in Répelin, 1920, J'ai également récolté un exemplaire de $L$. pradoanus de Verneuil et Lartet, 1863 dans un horizon ligniteux sous-jacent.

Le matériel étudié dans le niveau du Rognacien supérieur est beaucoup plus réduit que dans la série du calcaire de Rognac, il comprend: Palaeocyclophorus sp, Viviparus deshayesi Matheron, 1842, Bauxia bulimoides Matheron, 1832, et le genre Lychnus. Parmi ce dernier, seules les formes carénées sont présentes, le genre Lychnus ayant fait son apparition au Valdonien (Campanien) par l'espèce L. elongatus Roule, 1885, disparaitra complètement au-dessus du banc $\mathrm{n}^{\circ} 101$ (Fig. 2), les formes arrondies ne se récoltent que dans les assises du calcaire de la gare de Rognac qui est du Rognacien inférieur et également au sommet du Rognacien moyen (calcaire de Rognac).

Les gastéropodes fluvio-lacustres et continentaux sont mélangés par lessivage dans la partie sommitale du banc $\mathrm{n}^{\circ} 100$, celui-ci nous a fournis un échantillonnage de quatre espèces de Lychnus carénés qui sont:L. matheroni Requien, 1842, L. vitrollensis Répelin, 1920, L. siruguei nov. sp., et L. applanatus nov. sp. L'holotype du Lychnus siruguei nov. sp., reste dans l'ensemble en bon état avec la particularité de posséder encore un peu de coquille, il peut donc servir de diagnose dans le texte suivant, quant à l'holotype et le paratype du Lychnus applanatus nov. sp., malgré le bon état général des spécimens, les tours juvéniles restent érodés. 
G. Turin : BSGF 2017, 188, 38

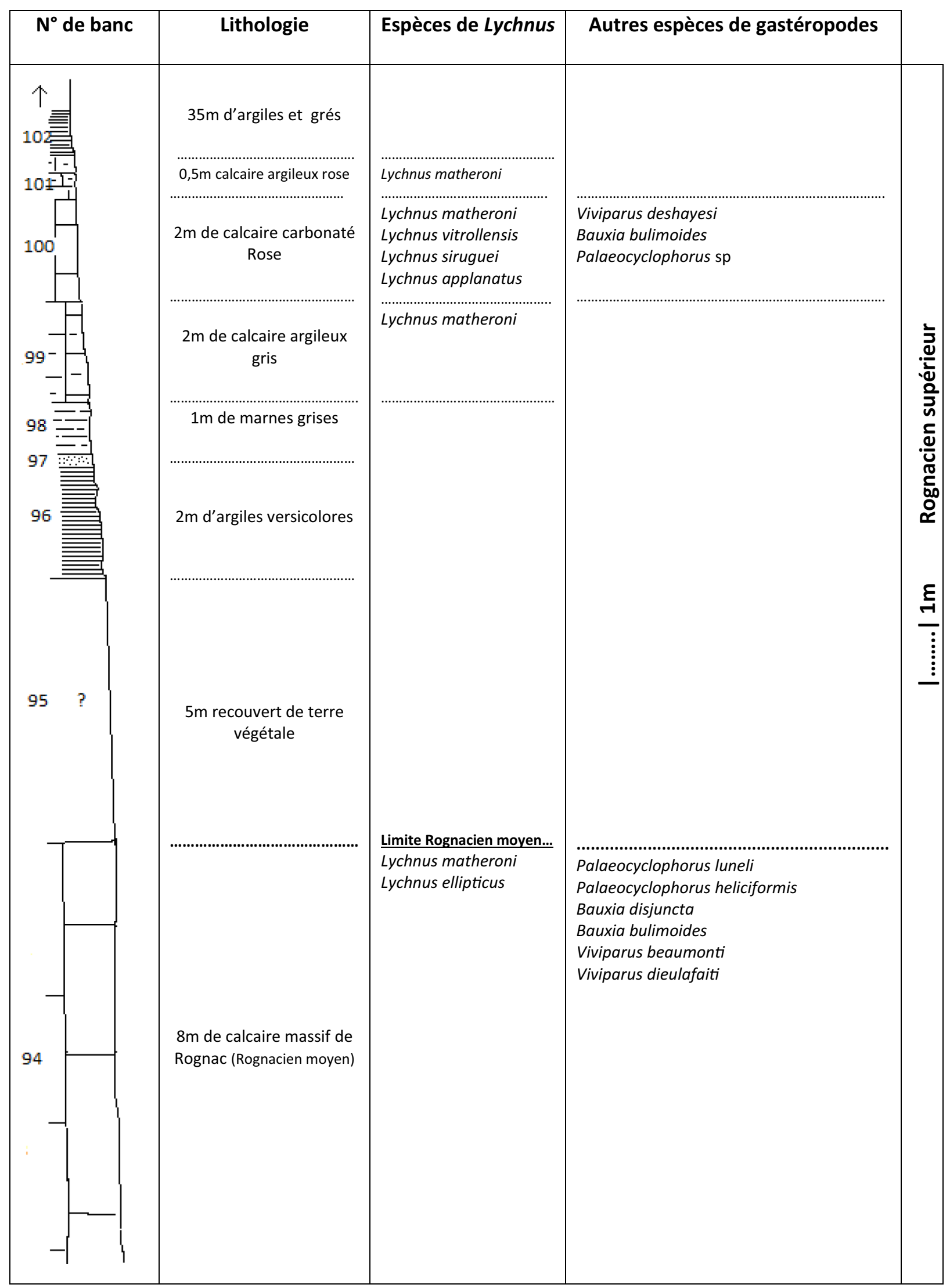

Fig. 2. Coupe A (Rognacien supérieur sous-jacent au radar de Vitrolles). 


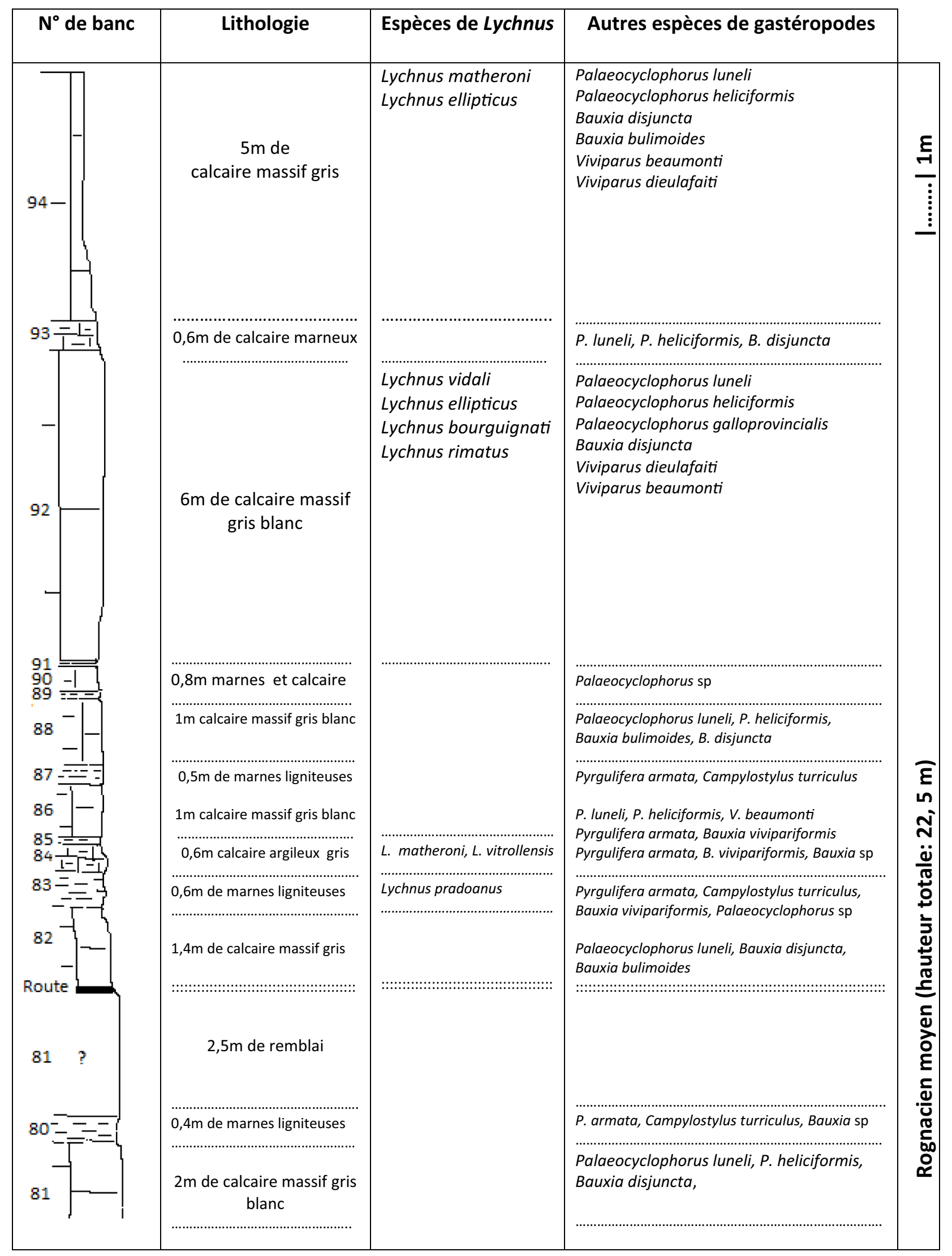

Fig. 3. Coupe B (Calcaire de Rognac sur la corniche du Roucas). 


\section{Systématique}

Famille : Anadromidae Wenz (1940)

Genre: Lychnus Matheron (1832)

Espèce-type: Lychnus ellipticus Matheron, 1832

Lychnus siruguei nov. sp. P1. 1, fig. $1 \& 2$.

Matériel type : Holotype MNHN.F.A59993 (Coll. Turin)

Paratype 1 spécimen (Coll. Turin)

Étymologie : l'espèce est dédiée à François Sirugue, ancien assistant du conservateur Raymond Dughi du Muséum d'Histoire Naturelle d'Aix-en-Provence.

Localité type: Vitrolles (Bouches-du-Rhône)

Étage : Rognacien supérieur (banc $n^{\circ} 100$, Fig. 2)

Description: Lychnus siruguei nov.sp., est une coquille elliptique, légèrement carénée, ovale dans le sens du diamètre transversal, elle comporte cinq tours de spire, dont les tours juvéniles sont dissimulés sur la moitié par le dernier. Le sens d'inclinaison de ces jeunes tours reste oblique par rapport à l'axe général de la coquille, la hauteur des deux premiers dépasse du reste de $2 \mathrm{~mm}$ l'épaisseur du tour final. La carène reste moins prononcée que sur les deux espèces voisines L. matheroni Requien et L. vitrollensis Répelin, (Répelin, 1920), elle est plutôt subarrondie et divise la coquille en deux parties. La face supérieure nous permet d'observer que le dernier tour est peu recouvrant et l'inférieure ou ombilicale qui possède une ligne suturale en forme de demi-circonférence. Sur cette face inférieure, la terminaison du dernier tour reste moins large près de l'ouverture que vers le milieu. La face supérieure est ornée de fines stries d'accroissement flexueuses, mais le reste du test est lisse. L'ouverture reste ovale, elle est ceinturée par un péristome légèrement réfléchi.

Dimensions: diamètre longitudinal, $33 \mathrm{~mm}$; diamètre transversal, $35 \mathrm{~mm}$; hauteur, $14 \mathrm{~mm}$; diamètre de l'ouverture, $15 \mathrm{~mm}$; hauteur de la forme jeune, $15 \mathrm{~mm}$.

Rapports et différences: Lychnus siruguei nov. sp., a une forme bien caractéristique et se distingue des autres espèces carénées par un diamètre transversal supérieur au diamètre longitudinal. Dans l'ensemble c'est à Lychnus matheroni Requien qu'il ressemble le plus mais ce dernier est beaucoup plus imposant et comporte quatre tours de spire au total (Requien, 1842 ; Fritel, 1903), alors que $L$. siruguei nov. sp., en comprend cinq, dont quatre au stade juvénile. Cette espèce est la seule dans le genre Lychnus à avoir une dimension transversale supérieure à la longitude, quant à $L$. vitrollensis Répelin, son diamètre longitudinal ne dépasse pas les $30 \mathrm{~mm}$ et sa coquille comporte seulement quatre tours de spire. À propos de cette dernière espèce, je partage les mêmes opinions que Fabre-Taxy, je considère que $L$. vitrollensis Répelin est une sous-espèce de L. matheroni Requien, (Fabre-Taxy, 1959), car ces deux espèces sont identiques à part les dimensions. Parmi toutes les espèces de ce genre, que ce soit des morphologies rondes ou carénées, Lychnus siruguei nov. sp., reste la seule qui comporte cinq tours pour une forme subovale. Un autre critère qui le différencie de $L$. matheroni Requien, reste l'aspect saillant dans le sens de la hauteur des deux premiers tours juvéniles. Les autres espèces provençales répertoriées dans le Rognacien, comprenant le même nombre de spires, sont les suivantes : $L$. dallonii Répelin, 1920, L. bourguignati Munier-Chalmas, 1885 et $L$. pradoanus Verneuil et Lartet, 1863. Lychnus dallonii Repelin comporte un sillon ombilical très long avec un diamètre longitudinal beaucoup plus important qui le différencie nettement de L. siruguei Nov.sp., L. bourguignati MunierChalmas, se définie par une forme arrondie sur sa face supérieure et aplati sur le côté ombilical, celui-ci a des dimensions beaucoup plus importantes et enfin L. pradoanus de Verneuil et Lartet qui comporte deux méplats circulaires autour de la carène.

Lychnus applanatus nov. sp. Pl. 1, fig. 3.

Matériel type : Holotype MNHN.F.A59994 (Coll. Turin) Paratype 1 spécimen (Coll. Turin)

Étymologie: la dénomination spécifique applanatus suggère la forme aplatie et étalée.

Localité type: Vitrolles (Bouches-du-Rhône)

Étage : Rognacien supérieur (banc $n^{\circ} 100$, Fig. 2)

Description: Lychnus applanatus nov. sp., est une forme allongée et très étalée longitudinalement, la carène reste assez bien prononcée, elle divise la coquille en deux parties. La face supérieure est bombée, la terminaison du dernier tour avant l'ouverture présente un plan très oblique par rapport à l'axe de la coquille, quant à la face ombilicale, elle reste très aplatie. Les deux spécimens récoltés, sont des moules internes, les tours juvéniles sont abrasés et ne permettent pas de déterminer leur nombre, dans l'ensemble, le dernier tour recouvre en épaisseur les premiers. La face inférieure très comprimée laisse apercevoir un sillon ombilical long et sinueux, le test reste lisse et les stries d'accroissement sont invisibles malgré la présence de coquille. L'ouverture reste ovale, elle est très écrasée sur la face ombilicale et ceinturée par un péristome réfléchi.

Dimensions: diamètre longitudinal, $50 \mathrm{~mm}$; diamètre transversal, $33 \mathrm{~mm}$; hauteur, $16 \mathrm{~mm}$; diamètre de l'ouverture longitudinale, $17 \mathrm{~mm}$; diamètre de l'ouverture transversale, $15 \mathrm{~mm}$.

Rapports et différences : Cette espèce a beaucoup d'affinité avec Lychnus elongatus Roule, 1885, (Repelin, 1920), mais s'en distingue par ses dimensions plus importantes. En ce qui concerne la position stratigraphique, L. elongatus Roule appartient à l'étage Valdonnien, (Campanien) alors que L. applanatus nov. sp., ne se récolte que dans le Rognacien supérieur. Toutes les autres espèces de ce genre que ce soit des morphologies carénées ou rondes n'ont pas de ressemblance proche avec cette nouvelle forme.

\section{Répartition}

Ces deux nouvelles espèces proviennent uniquement du niveau calcaire du Rognacien supérieur de Vitrolles (banc $n^{\circ}$ 100, Fig. 2), elles sont représentées chacune sur cette localité type par deux exemplaires. Avec Jean-Luc Martin, nous avons suivi cet horizon, au nord à Saragousse sur la commune de Rognac et au sud sur le Griffon, je précise qu'en dépit de plusieurs années de recherches, nous n'y avons jamais trouvé ni L. siruguei nov. sp., et L. applanatus nov. sp.

Les deux autres espèces, $L$. matheroni Requien, et L. vitrollensis Répelin recensées également dans ce banc $n^{\circ}$ 100 , sont présentes stratigraphiquement dans les horizons ligniteux intermédiaire au calcaire de Rognac, personnellement j'ai récolté ces deux espèces dans le niveau $\mathrm{n}^{\circ} 84$ de ce calcaire, au niveau de la corniche du Roucas (Fig. 3).

Nous n'avons pas négligé également avec Jean-Luc Martin, le calcaire de Rognac et ses niveaux ligniteux de la commune des Pennes-Mirabeau. Nos reconnaissances ont été poussées à la sortie du tunnel de l'autoroute $\mathrm{A} 7$ et nous avons récoltés dans 


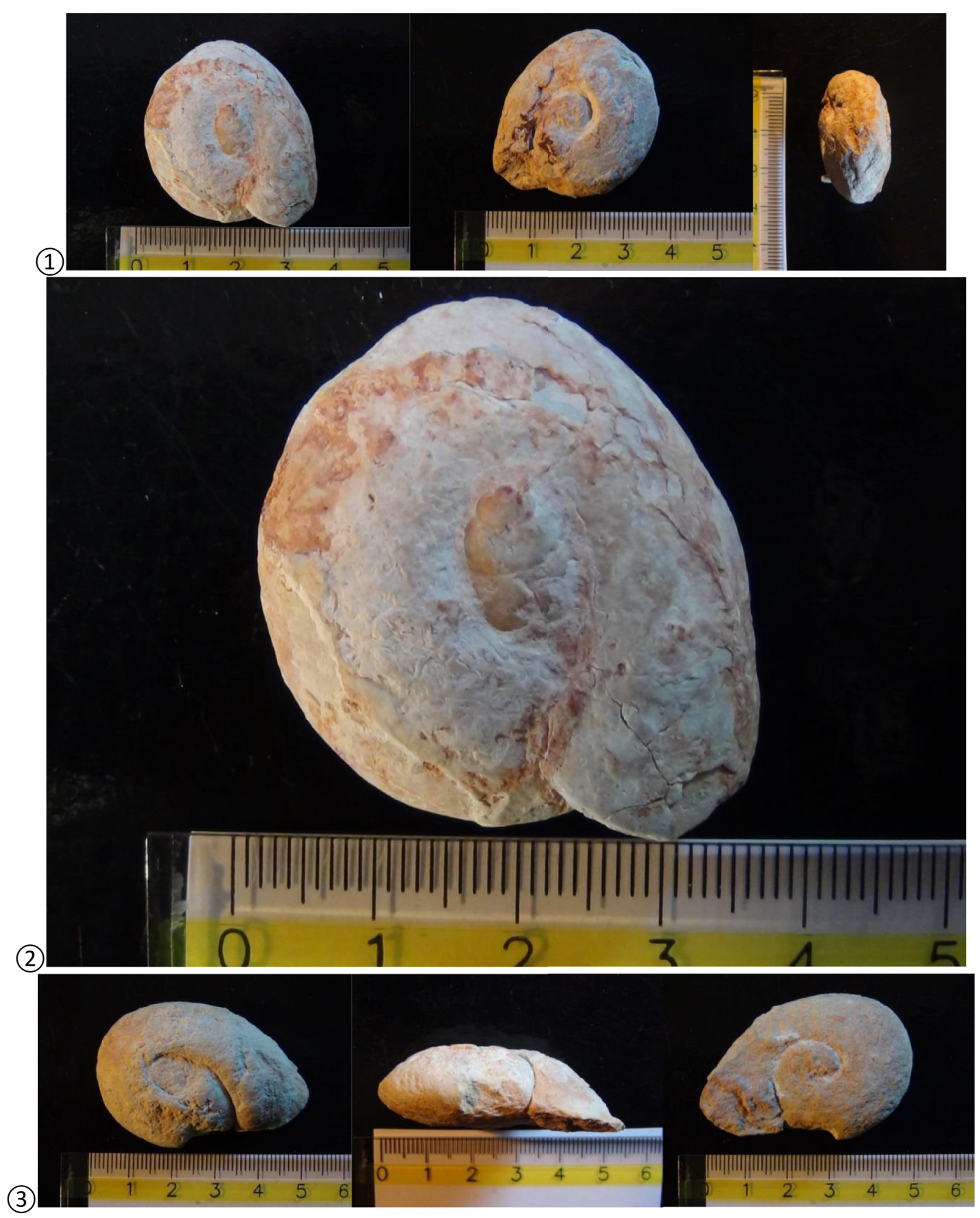

Planche 1

Lychnus provenant du Rognacien supérieur de Vitrolles, niveau 100 de la coupe A.

Fig. 1. Lychnus siruguei nov.sp., holotype, MNHN.F.A59993 (Collection Turin).

Fig. 2. Lychnus siruguei nov. sp., holotype, MNHN.F.A59993 (Coll. Turin). X 2,5

Fig. 3. Lychnus applanatus nov. sp., holotype, MNHN.F.A59994 (Coll. Turin).

\section{Plate 1}

Lychnus from the upper Rognacian Vitrolles, level 100 of section A.

Fig. 1 Lychnus siruguei nov. sp., holotype, MNHN.F.A59993 (Turin collection).

Fig. 2 Lychnus siruguei nov. sp., holotype, MNHN.F.A59993 (Turin collection). X 2,5

Fig. 3 Lychnus applanatus nov. sp,. holotype, MNHN.F.A59994 (Turin collection) 
un banc marneux de ce Rognacien moyen, un exemplaire de Lychnus matheroni Requien, accompagné d'un magnifique spécimen de Lychnus cf. gardanensis Matheron ${ }^{1}$.

\section{Conclusion}

Les espèces Lychnus siruguei nov. sp., et L. applanatus nov. sp., apparaissent à Vitrolles au sommet du banc $\mathrm{n}^{\circ} 100 \mathrm{du}$ Rognacien supérieur. Elles coexistent avec L. matheroni Requien et $L$. vitrollensis Répelin avant de disparaître complètement avec les autres gastéropodes dans les niveaux d'argiles et calcaire marneux sus-jacent qui sont encore Rognacien. Seule l'espèce $L$. matheroni a été encore recensée récemment par moi-même, dans le banc $n^{\circ} 101$ (Fig. 2). Ce taxon sera le dernier représentant du genre, l'extinction définitive des Lychnus sera configurée dans la zone supérieure (niveau $\mathrm{n}^{\circ} 102$ ).

La découverte de ces nouvelles espèces rarissimes qui sont peut-être des taxons endémiques, renforce l'originalité paléobiogéographique des faunes de Lychnus de Provence. Pour la même période, ce genre qui est également présent en Espagne est moins diversifié.

En recensant ces deux nouvelles formes, ce sont treize espèces du genre Lychnus qui sont désormais connues dans le Rognacien des Bouches-du-Rhône et quinze au total en ajoutant les sous étages Bégudien et Valdonnien.

\section{Références}

d'Orbigny A. 1850. Prodome de Paléontologie stratigraphique universelle des animaux mollusques et rayonnés. Paris: Masson, 2, $298 \mathrm{p}$.
Dughi R, Sirugue F. 1958. Sur les œufs de dinosaures du bassin fluviolacustre de Basse-Provence. Section des sciences, $83^{\circ}$ congrès des sociétés savantes, $192 \mathrm{p}$.

Durand JP. 1978. Notice explicative de la Feuille de MartiguesMarseille à 1/50 000. Orléans : Édition du Bureau de Recherches Géologiques et Minières, pp. 1-51.

Fabre-Taxy S. 1959. Faunes lagunaires et continentales du Crétacé supérieur de Provence. Le Maestrichtien et le Danien. Annales de Paléontologie 45: 55-124.

Fritel P-H. 1903. Histoire Nat. de la France, $24^{\circ}$ partie, Paléontologie. Paris : Deyrolle, 229 p.

Haug E. (1908-1911). Traité de Géologie. Paris : A. Colin, 2, 1410 p.

Matheron P. 1832. Observations sur les terrains tertiaires des Bouches-du-Rhône et description des coquilles fossiles inédites ou peu connus qu'ils renferment. Annales des Sciences et Industrie du Midi de la France 3: 87 p.

Matheron P. 1843. Catalogue méthodique et descriptif des corps organisés fossiles du département des Bouches-du-Rhône et lieux circonvoisins. Répertoire des Travaux de la Société de Statistique de Marseille 6(81-342): 1-41.

Matheron P. 1862. Recherches comparatives sur les dépôts fluviolacustres tertiaires des environs de Montpellier, de l'Aude et de la Provence. Marseille: Arnaud \& Cie, 112 p.

Repelin J. 1899. Catalogue méthodique détaillé de la collection paléontologique de Philippe Matheron. Marseille: Saint-Léon, $146 \mathrm{p}$.

Repelin J. 1920. Monographie du genre Lychnus. Mémoires de la société géologique de France. Paléontologie, Mémoire ${ }^{\circ} 5323$ (524): 1-6.

Requien E. 1842. Description du Lychnus matheroni. Bulletin de la Société géologique de France 13: 495-496.

Roule L. 1885. Description de quelques coquilles fossiles du calcaire lacustre de Rognac. (Bouches-du-Rhône). Bulletin de la Société Malacologique 1: 311-326.

Citation de l'article : Turin G. 2017. Description de deux espèces nouvelles du genre Lychnus (Mollusca, Gastropoda, Anadromidae) au Rognacien (Maastrichtien supérieur) de Vitrolles (Bouches-du-Rhône, France), Bull. Soc. géol. Fr. 188: 38.

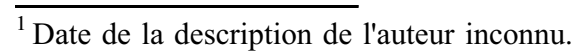

